\title{
Mediastinal Rhabdomyosarcoma Feeding Off the Left Anterior Descending Artery
}

\author{
Milena Guenther ${ }^{1}$ Markus Richter ${ }^{1} \quad$ Torsten Doenst $^{1} \quad$ Tobias Rachow $^{2}$ Susanne Lang ${ }^{3}$ Tim Sandhaus ${ }^{1}$
}

1 Department of Cardiothoracic Surgery, Jena University Hospital, Jena, Thüringen, Germany

2 Department of Pneumology, Jena University Hospital, Jena, Thüringen, Germany

${ }^{3}$ Department of Hematology and Internal Oncology, Jena University Hospital, Jena, Thüringen, Germany

Thorac Cardiovasc Surg Rep 2022;11:e17-e19.
Address for correspondence Torsten Doenst, MD, PhD., Department of Cardiothoracic Surgery, Jena University Hospital, Am Klinikum 1, 07747 Jena, Germany (e-mail: doenst@med.uni-jena.de).

\begin{abstract}
Keywords

- mediastinal rhabdomyosarcoma

- pericardium

- off-pump stabilizer

- surgery

- complication

We report the rare case of a 51-year-old patient with a $15 \mathrm{~cm}$ mediastinal rhabdomyosarcoma with blood supply from the left anterior descending artery presenting as a large mass including the pericardium with extensive contact to the epicardium compressing heart and left lung. The tumor was successfully removed through median sternotomy, blunt dissection from the heart and the left lung, resection of the infiltrated pericardium, and ligation of the tumor-feeding vessels using off-pump stabilizers. Histopathological examination revealed a spindle cell rhabdomyosarcoma with $\mathrm{R} 0$ resection. The postoperative course was uneventful, and patient is feeling well at 3-month follow-up.
\end{abstract}

\section{Introduction}

We report the rare case of a 51-year-old patient with a $15 \mathrm{~cm}$ mediastinal rhabdomyosarcoma (RMS) with blood supply from the left anterior descending artery (LAD) presenting as a large mass including the pericardium with extensive contact to the epicardium compressing heart and left lung. The tumor was successfully removed through median sternotomy, blunt dissection from the heart and the left lung, resection of the infiltrated pericardium, and ligation of the tumor-feeding vessels using off-pump stabilizers. Histopathological examination revealed a spindle cell RMS with R0 resection. The postoperative course was uneventful, and patient is feeling well at 3-month follow-up. received

December 23, 2020 accepted after revision March 8, 2021

\section{Case Description}

A 51-year-old patient was admitted to the hospital due to dyspnea at rest, dry cough, and increasing peripheral edema. At the age of 20, the patient was diagnosed with an embryonic RMS in the left leg, which was successfully treated by amputation. Now, echocardiography revealed hemodynamically relevant hemorrhagic pericardial effusion, which was drained by pericardiocentesis $(1,400 \mathrm{~mL})$. CT scan showed a large mass including the pericardium with extensive contact to the epicardium compressing heart and left lung. MRI confirmed the suspicion of a solid mass without visible signs of myocardial or lung tissue infiltration ( $\mathbf{- F i g . 1 A}$ ). Coronary angiography showed arterial vessels originating from the LAD supplying the tumor ( - Fig. 1B). Multidisciplinary tumor
DOI https://doi.org/ 10.1055/s-0041-1729928. ISSN 2194-7635.

\footnotetext{
(C) 2022. The Author(s).

This is an open access article published by Thieme under the terms of the Creative Commons Attribution-NonDerivative-NonCommercial-License, permitting copying and reproduction so long as the original work is given appropriate credit. Contents may not be used for commercial purposes, or adapted, remixed, transformed or built upon. (https://creativecommons.org/ licenses/by-nc-nd/4.0/) Georg Thieme Verlag KG, Rüdigerstraße 14, 70469 Stuttgart, Germany
} 
A

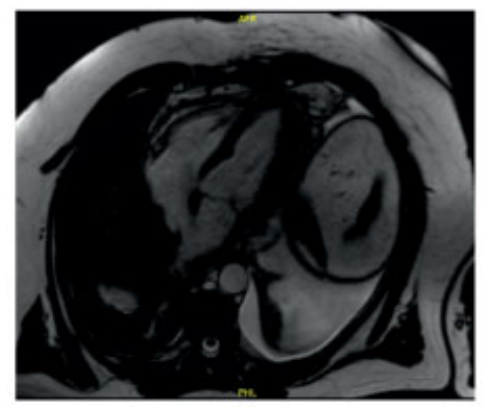

B

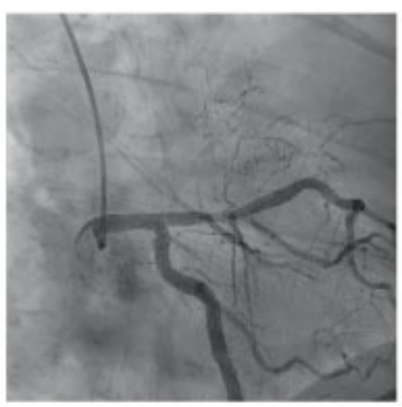

C

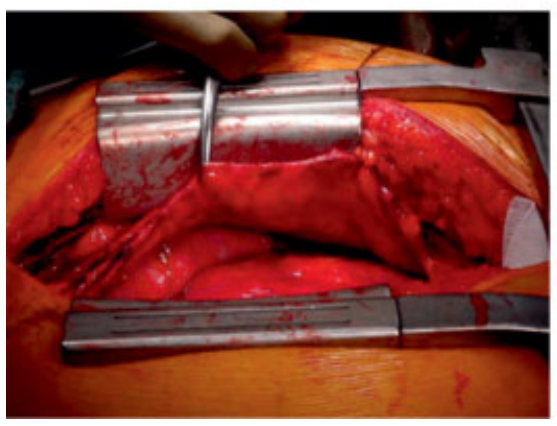

D

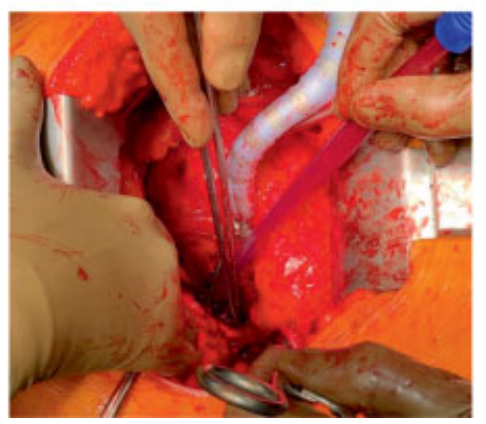

E

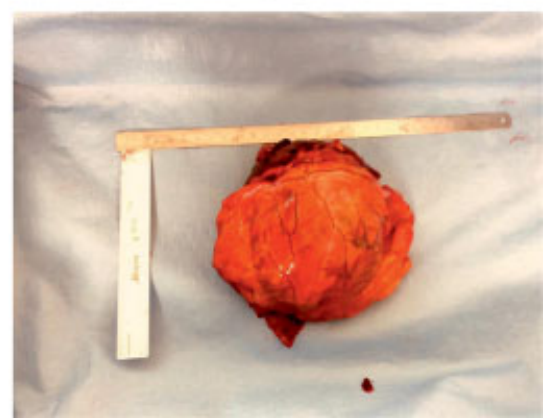

Fig. 1 Panels A, B: Diagnostics via MRI and coronary angiography. (A) MRI of the solid tumor in the left hemithorax excluding visible signs of myocardial or lung tissue infiltration. (B) Coronary angiography demonstrating arterial vessels originating from the LAD supplying the tumor. Panels C, E: Intraoperative photographs. (C) The tumor after median sternotomy before dissection from the heart. Pericardial infiltration can be seen on the left side underneath the pericardial edges elevated with the forceps. (D) Stabilized LAD territory showing the tumor feeding vessels. (E) In toto resected tumor. LAD, left anterior descending artery; MRI, magnetic resonance imaging.

board reached the decision for surgical removal. Median sternotomy revealed a $15 \mathrm{~cm}$ mass located between the heart and the left lung with pericardial infiltration. The tumor was dissected from the left lung without the need for lung tissue resection (-Fig. 1C). Blunt dissection from the heart up to the coronary vessels feeding the tumor was also possible. Using off-pump stabilizers, the tumor-feeding vessels could be ligated, and resection completed without affecting perfusion of the LAD ( - Fig. 1D and E). The pericardium was generously resected and reconstructed with a bovine patch. Histopathological examination revealed the morphology of a spindle cell variant of RMS (G2: intermediate grade) with negative resection margins (R0). The patient was extubated at the first day postoperatively and further recovery was uneventful. At 3-month follow-up examination the patient was in good general condition. Positron emission tomography/ computed tomography scan at 4 months showed no vital tumor tissue.

\section{Discussion}

To the best of our knowledge, this case is the first description of a RMS with blood supply originating from the LAD. By combining cardio- and oncosurgical techniques a complete resection of this complex tumor could be achieved, potentially providing the best prognosis.

RMS are the most common malignant tumors of soft, muscle tissue in young adults. They are subdivided into three major groups: embryonal, alveolar, and pleomorphic, due to their distinct clinical and prognostic features. Embryonal RMS can occur anywhere in the body, but some localization such as head, neck, and urogenital tract are primarily affected. The general prognosis of these tumors is usually intermediate. After chemotherapy, surgery, and radiation, a 65 to $75 \%$ 5-year survival rate has been reported. ${ }^{1}$ Pleomorphic and alveolar RMS are more aggressive with 5-year survival rates ranging between 20 and $30 \%{ }^{1}$ They occur mostly in skeletal muscles of extremities. ${ }^{1}$ Histopathological examination of our patient revealed a spindle cell variant of RMS. Although this tumor represents a subgroup of the embryonal RMS, it is characterized by a more favorable prognosis (5-year survival rate of approximately 95\%). ${ }^{1}$ However, in our case we found a potential metachronous distant manifestation of a tumor originally located in the leg, which makes the prediction of the true prognosis difficult. Tumor recurrence has been reported in 30 to $40 \%$ of all cases, but most of them occur within the first year and are associated with the involvement of lymph nodes, unfavorable tumor histology, and localization. ${ }^{2}$ In our patient, the tumor was identified 30 years after identification of the first RMS. Since the histological assessment 30 years ago differed from today, ${ }^{3}$ it is impossible to determine with certainty whether the two RMS arose from the same original tumor tissue. Thus, it remains unclear if the two tumors are linked and we hope for a favorable prognosis in our patient.

While tumors of the heart are extremely rare, RMS account for almost $20 \%$ of all primary malignant neoplasms 
of the heart. ${ }^{4}$ They usually arise from the ventricular walls, are highly lethal, and present with varying symptoms. Castorino et $\mathrm{al}^{4}$ reported a case with a tumor arising from the atrial wall and presented as severe mitral stenosis. Emergency surgery was required. Moreover, RMS of the right ventricle presenting as chest pain and leading to congestive heart failure is reported by Nagata et al in $1982 .{ }^{5}$ In our case, the tumor was located between heart and left lung presenting as hemodynamically relevant hemorrhagic pericardial effusion. The tumor was also unique in its blood supply. Vessels of substantial size originated from the LAD. Resection therefore jeopardized perfusion of the anterior wall of the heart, and myocardial infarction was therefore a potential complication in this case. Despite the high lethality of tumors in this special localization and the increased risk of relapse in our patient a complete resection of the tumor could be achieved, and he is free from tumor at 4 months postoperatively supporting our assumption for a good prognosis.

The literature contains one similar case of a tumor supplied by a coronary artery. It describes the case of a 20-yearold-woman who presented with retrosternal pain, peripheral edema, and pericardial effusion. The effusion was drained, and a surgical biopsy revealed a pleomorphic RMS. The authors reported an invasive growth into the ventricle and surgery was not performed. The patient was transferred to receive chemotherapy. ${ }^{6}$ Follow-up was not reported, but the treatment concept may not have been curative. Our case shows that complex tumors such as this RMS can be successfully treated by surgical resection.

Surgical therapy forms the essential base of the treatment of soft tissue sarcomas. Histopathological examination revealed an intermediate grade (G2) RMS with R0 resection. Since soft tissue sarcomas are only moderately sensitive to radiation, therapy is only indicated in patients with incomplete resection, higher grading (G3-G4), and palliative situation. Chemotherapy has been shown to be effective in children. In adults the effect of chemotherapy remains unclear. Adjuvant chemotherapy can be considered in patients with higher grade (G3-G4) soft tissue sarcomas, preferred under study conditions. As the tumor in our patient was intermediate grade (G2) adjuvant radiation and chemotherapy was not indicated. ${ }^{7}$

Surgical removal may be challenging, which is mainly due to the potential invasiveness and the unique blood supply from a coronary artery. Based on our routine use of off-pump coronary surgery techniques, we could stabilize the beating heart, and carefully resect the tumor until the blood vessels feeding the tumor were identified. They could then be ligated using 4-0 Prolene sutures and the tumor was resected in toto. The extensive imaging performed preoperatively paid off in that we used the optimal approach in this case. A leftsided thoracotomy would have prevented us from identifying the blood vessels originating from the LAD. This case is therefore a prime example for the advantage of combining cardio- and oncosurgical techniques, specifically the combination of median sternotomy, off-pump coronary surgery tools, and oncosurgical resection.

\section{Conclusion}

This case is the first description of a RMS with blood supply from the LAD. Combining cardio- and oncosurgical techniques allowed a complete resection of this complex tumor, potentially providing the best prognosis.

Conflict of Interest

None declared.

\section{References}

1 Schlag PM, Hartmann JT, Budach V. Weichgewebetumoren: Interdisziplinäres Management. Berlin, Heidelberg: Springer-Verlag; 2011;15;

2 Bisogno G, De Salvo GL, Bergeron C, et al. Maintenance low-dose chemotherapy in patients with high-risk (HR) rhabdomyosarcoma (RMS): a report from the European Paediatric Soft Tissue Sarcoma Study Group (EpSSG). J Clin Oncol 2018;36;

3 Caillaud JM, Gérard-Marchant R, Marsden HB, et al. Histopathological classification of childhood rhabdomyosarcoma: a report from the International Society of Pediatric Oncology pathology panel. Med Pediatr Oncol 1989;17(05):391-400

4 Castorino F, Masiello P, Quattrocchi E, Di Benedetto G. Primary cardiac rhabdomyosarcoma of the left atrium: an unusual presentation. Tex Heart Inst J 2000;27(02):206-208

5 Nagata K, Irie K, Morimatsu M, Nakashima T, Eriguchi N, Koga M. Rhabdomyosarcoma of the right ventricle. Acta Pathol Jpn 1982; 32(05):843-849

6 Villacampa VM, Villarreal M, Ros LH, Alvarez R, Cózar M, Fuertes MI. Cardiac rhabdomyosarcoma: diagnosis by MR imaging. Eur Radiol 1999;9(04):634-637

7 Adjuvant chemotherapy for localised resectable soft tissue sarcoma in adults. Sarcoma Meta-analysis Collaboration (SMAC) Cochrane Database Syst Rev 2000;(02):CD001419 